Service social

\title{
Les attitudes des parents à l'égard de l'informatique et leur appréciation de l'utilisation du logiciel Vie familiale et impact de la maladie
}

\author{
Martin Poulin, André Beaudoin et Françoise Turgeon-Krawczuk
}

Volume 43, numéro 2, 1994

Enfants, parents, intervenants

URI : https://id.erudit.org/iderudit/706658ar

DOI : https://doi.org/10.7202/706658ar

Aller au sommaire du numéro

Éditeur(s)

École de service social de l'Université Laval

ISSN

1708-1734 (numérique)

Découvrir la revue

Citer cet article

Poulin, M., Beaudoin, A. \& Turgeon-Krawczuk, F. (1994). Les attitudes des parents à l'égard de l'informatique et leur appréciation de l'utilisation du logiciel Vie familiale et impact de la maladie. Service social, 43(2), 81-96. https://doi.org/10.7202/706658ar
Résumé de l'article

Le logiciel Vie familiale et impact de la maladie intègre quatre tests adaptés pour l'intervention en service social et donne une rétroaction immédiate à l'utilisateur sur dix résultats présélectionnés. Cet article décrit

1) les attitudes des parents d'enfants atteints d'une maladie chronique à l'égard de l'utilisation de l'ordinateur comme support à l'intervention clinique et 2) leurs réactions à la suite de l'utilisation interactive du logiciel. Même lorsqu'ils ont peu ou pas d'expérience avec les ordinateurs, les parents font une évaluation positive du programme Vie familiale, quels que soient leur âge, leur sexe ou leur degré d'instruction. 


\title{
Les attitudes des parents à l'égard de l'informatique et leur appréciation de l'utilisation du logiciel Vie familiale et impact de la maladie
}

\author{
Martin POULIN \\ Professeur titulaire \\ École de service social, Université Laval \\ André BEAUDOIN \\ Professeur titulaire \\ École de service social, Université Laval \\ Françoise TURGEON-KRAWCZUK \\ Chercheure, Hôpital Rivière-des-Prairies, Montréal
}

Plusieurs recherches ont montré que les enfants aux prises avec une maladie évolutive à issue fatale ainsi que leur famille présentent un risque élevé de problèmes psychologiques et sociaux (Cadman et al., 1987). Les parents doivent apprendre à vivre avec un stress chronique et ils ont à se mobiliser pour donner des traitements quotidiens tout en maintenant une vie familiale aussi normale que possible. Ils ont aussi à s'adapter rapidement à un milieu hospitalier fort complexe: 
ils doivent parfois prendre des décisions difficiles concernant les interventions et les traitements médicaux prescrits pour leur enfant. Dans un tel contexte, I'approche biopsychosociale utilisée par les équipes de soins est essentielle dans la prise en charge de la maladie. L'intervention du service social, intégrée à cette approche, vise à soutenir les parents, à les aider à comprendre et à interpréter une situation complexe, à augmenter leur compétence au moyen de stratégies d'adaptation, tout en améliorant leurs relations familiales et sociales.

Le développement du logiciel interactif Vie familiale et impact de la maladie se situe dans la suite de la démarche d'informatisation des données à la clinique de fibrose kystique de l'hôpital Sainte-Justine, qui vise à favoriser l'approche biopsychosociale. Le logiciel Vie familiale et impact de la maladie se compose de quatre tests :

- la mesure de stress psychologique (Lemyre-Tessier, 1986);

- I'index de stress parental (Abidin, 1983);

- I'échelle du climat familial (Moos, 1974);

- le questionnaire d'adaptation à la vie de couple (Locke-Wallace,1959).

Le lecteur peut trouver ailleurs une description du contenu du logiciel'. Celui-ci constitue un instrument d'intervention du service social dont un des objectifs principaux est d'améliorer la compétence du parent. L'interprétation immédiate des résultats faite au parent lui révèle quels sont ses points forts et ses points faibles par rapport aux dix dimensions qui font partie de l'interprétation. Ce dernier est ainsi en mesure de porter attention aux points plus faibles et de consulter un professionnel lorsque cela est nécessaire.

Le présent article livre une partie des résultats contenus dans une recherche plus globale portant sur les effets et l'appréciation (TurgeonKrawczuk, 1991; Poulin et al., 1991) de l'utilisation du logiciel interactif sur la compétence des parents d'enfants atteints de fibrose kystique, suivis dans les cliniques spécialisées où ils sont les bénéficiaires des

1. Il a été décrit dans un article paru dans la revue Service social (vol. 41, no 3 ) intitulé "État des connaissances à l'origine de I'utilisation du logiciel Vie familiale et impact de la maladie : attitudes et motivation de la clientèle et préoccupations des professionnels » (Poulin et al., 1992: 116 et 117). Ce logiciel, d'usage facile, permet au parent d'effectuer les tests directement à l'ordinateur, de manière interactive, selon une procédure très simple et suivant les directives qui apparaissent à l'écran. Une partie des résultats des tests est présentée à l'écran sous forme de graphiques accompagnés d'une interprétation rendue immédiatement accessible au répondant sans l'intermédiaire d'un intervenant. Les résultats écrits remis au parent ne contiennent que les interprétations sans les graphiques. Les tests sont répétés trois fois, ce qui permet à l'utilisateur de comparer ses résultats d'une fois à l'autre. 
interventions du service social. Les données ont été recueillies à la suite de l'utilisation du logiciel à trois reprises sur une période de 12 mois, entre novembre 1989 et octobre 1990. Le contenu de l'article vise à répondre à la question suivante: Quelles sont les attitudes des parents à l'égard de l'usage de l'informatique comme appui à l'intervention du service social et quelle appréciation les parents font-ils du logiciel qui leur est offert? L'obtention du point de vue des parents sur la technologie informatique et sur le contenu de l'évaluation qu'elle rend possible est essentielle pour une meilleure connaissance des avantages et des limites d'une telle stratégie d'intervention.

\section{MODĖLE D'ÉTUDE DES ATTITUDES ET DE L'APPRÉCIATION DES PARENTS}

La recension des écrits (Poulin et al., 1992: 105-115) sur les applications cliniques de l'informatique impliquant directement la clientèle a permis de déterminer les éléments à retenir en ce qui a trait à l'exploration des attitudes de résistance et d'attente à l'égard de I'utilisation de l'informatique. Il se dégage de ces écrits un certain nombre de constatations : bonne acceptation des tests auto-administrés par la clientèle (Johnson et Williams, 1980 ; Erdman et al., 1981 ; Erdman et Foster, 1986; Hedlund et al., 1985 ; Mathisen, 1988); résistance encore forte des cliniciens (Mathisen, 1988) qui soulèvent des problèmes de confidentialité (Mezzich et al., 1982 ; Sampson, 1983b; Merrell, 1985), de déshumanisation (Space, 1981 ; Sampson, 1983b) et de standardisation (Space, 1981 ; Erdman et al., 1985). La connaissance des effets sur la clientèle de l'utilisation de l'informatique est par ailleurs limitée, la recherche évaluative dans ce domaine en étant à ses débuts (Siegel et Alexander, 1988 ; Mathisen, 1988). Certaines caractéristiques personnelles de I'utilisateur influencent ces effets: I'âge, le sexe, la scolarité (Skinner et Steinhauer, 1983) et la connaissance de l'informatique (Sampson, 1983a; Johnson et al., 1981 ; Merrell, 1985 ; Hofer, 1985).

En continuité avec les travaux d'auteurs américains qui ont constaté la réaction favorable de la clientèle aux tests interactifs (Hedlund, 1988; Mathisen, 1988), les résistances et attentes, de même que I'appréciation des parents utilisateurs du logiciel interactif, sont mises en relation avec un certain nombre de variables. Les caractéristiques retenues comme variables indépendantes des attitudes (résistances et attentes) et de l'appréciation de l'utilisation du logiciel sont l'âge, le sexe, la scolarité et le degré de contact avec l'informatique des répondants. Le schéma de la page suivante résume ces relations. 
Variables indépendantes

\begin{tabular}{|l|}
\hline Âge \\
Sexe
\end{tabular}

Degré initial de contact avec l'informatique
Variables dépendantes

Résistance et attentes

face à I'utilisation

de l'informatique

Appréciation

de I'utilisation

du logiciel

\section{Les caractéristiques des parents utilisateurs du logiciel}

Au début de la recherche, le groupe se compose de 43 parents, dont la moyenne d'âge est de 33,9 ans. La moyenne d'âge des 29 mères est de 32,4 ans et celle des 14 pères de 37 ans. Des 43 parents, 26 ont une scolarité plutôt faible (études primaires et secondaires) et 17 ont une scolarité élevée (études collégiales et universitaires). Le groupe final de répondants, composé de 40 parents en raison de trois abandons (deux mères et un père) en cours d'expérience, conserve les mêmes caractéristiques qu'au début de l'expérimentation.

\section{Le degré de contact avec l'informatique}

La compétence plus ou moins grande de la personne dans I'utilisation de l'ordinateur est un facteur mis en évidence dans plusieurs études en relation avec les attitudes et la satisfaction de la clientèle (Johnson et al., 1981 ; Sampson, 1983a; Merrell, 1985 ; Hofer et Green, 1985). Nous adaptons à notre situation de recherche le questionnaire mis au point par Béliveau et Poulin (1987) pour mesurer le degré initial de contact des parents avec l'informatique à partir d'un indice énumératif. Cet indice provient de réponses données à sept questions portant sur trois dimensions: la formation en informatique; les contacts avec l'informatique à la maison; et les contacts avec l'informatique au travail. Le degré de contact est proportionnel au nombre de réponses positives indiquant une forme de contact avec l'informatique. II se mesure par la somme des scores obtenus à l'ensemble des questions. Un score élevé indique un plus haut degré de contact avec l'informatique.

Le degré de contact des répondants avec l'informatique est établi immédiatement après la première utilisation du logiciel. En ce qui a trait à la première dimension, la formation, les résultats sont les suivants : un seul parent a bénéficié d'un programme complet de formation en informatique, alors que huit autres $(20 \%)$ ont suivi quelques cours dans 
le domaine; les autres répondants $(77,5 \%)$ n'ont pas de formation particulière. Concernant la deuxième dimension, celle des contacts avec l'informatique à la maison, 32 répondants ( $80 \%)$ disent n'avoir aucun contact. Sur les huit répondants $(20 \%)$ qui disposent d'un ordinateur à domicile, deux l'utilisent régulièrement, cinq s'en servent à I'occasion et un ne s'en sert jamais. Enfin, relativement à la troisième dimension des contacts avec l'informatique au travail, la majorité des répondants $(65,9 \%)$ disent ne jamais se servir d'un ordinateur.

Le degré de contact avec l'informatique se mesure par la somme des scores obtenus à l'ensemble des questions de l'échelle (pour les trois dimensions) et seules les personnes ayant répondu à toutes les questions $(n=31)$ sont considérées dans le calcul du score total. Sur le plan de la consistance interne, l'analyse produit un coefficient alpha de Cronbach de 0,897. Les scores varient entre 5 et 17 avec une moyenne de 8,25 et un écart-type de 3,99. Sont considérés comme ayant un degré de contact faible les répondants qui cumulent de 5 à 8 points; un degré moyen, ceux qui obtiennent de 9 à 13 points; et élevé, ceux qui se situent entre 14 et 17 points.

Des 20 parents $(64,5 \%)$ qui ont un degré de contact faible, 14 n'ont aucun contact avec l'informatique. Le nombre de parents ayant un degré de contact élevé est de six, alors que cinq ont un degré de contact moyen. Les mères possèdent un degré de contact qui équivaut à celui des pères (voir tableau 1). II ressort de ces données que la majorité des répondants, pères aussi bien que mères, ne connaissent pas l'informatique.

\section{Tableau 1}

\section{Degré de contact avec l'informatique pour les parents, pères et mères}

\begin{tabular}{lrrrrrrrr}
\hline Répondants & \multicolumn{2}{c}{ Faible } & \multicolumn{2}{c}{ Moyen } & \multicolumn{2}{c}{ Élevé } & \multicolumn{2}{c}{ Total } \\
& $\mathrm{n}$ & $\%$ & $\mathrm{n}$ & $\%$ & $\mathrm{n}$ & $\%$ & $\mathrm{n}$ & $\%$ \\
Parents* & 20 & 64,5 & 5 & 16,1 & 6 & 19,3 & 31 & 100 \\
Pères & 7 & 63,6 & 2 & 18,1 & 2 & 18,1 & 11 & 100 \\
Mères & 13 & 65,0 & 3 & 15,0 & 4 & 20,0 & 20 & 100 \\
\hline
\end{tabular}

* Parents ayant répondu à toutes les questions.

\section{LES ATTITUDES À L'ÉGARD DE L'INFORMATIQUE}

La résistance se définit comme étant "une tendance à freiner la nouveauté » (Grawitz, 1986, p. 322) et l'attente comme un "état d'esprit subjectif, influencé par la capacité d'ajustement de l'individu à la 
situation à venir qu'il anticipe, souvent en fonction de son expérience passée » (p. 28). Les auteurs consultés proposent différentes façons de mesurer les résistances et attentes: évaluation par un observateur (Logie et al., 1976); questionnaire (Klinger et al., 1976); entrevue (Costello, 1982); utilisation d'une échelle d'attitudes (Lucas, 1977 ; Skinner et Allen, 1983 ; Carr et Ghosh, 1983).

Nous retenons l'échelle de Lucas (1977), construite pour mesurer les attitudes des patients suivis dans une clinique médicale à l'égard de I'interrogation par ordinateur; cette échelle peut s'adapter aux parents d'enfants malades suivis dans une clinique spécialisée par une équipe pluridisciplinaire. Deux équipes indépendantes composées chacune de deux membres ont procédé à une traduction inversée des 22 items inclus dans l'échelle de Lucas. La traduction française retenue a ensuite été élargie dans son application, du domaine médical au domaine psychosocial, en remplaçant le mot «patient» par le mot "bénéficiaire» et le terme "médecin » par le concept d'équipe.

L'échelle d'attitudes est remplie par les parents immédiatement après leur première utilisation du logiciel. Les réponses aux 22 énoncés sont mesurées par une échelle de Likert à six points. Le tableau en annexe indique les attitudes des parents à l'égard de l'informatique en relation avec chaque item de l'échelle. Les résultats ont été regroupés de la façon suivante: le nombre des personnes défavorables est établi par l'addition des fréquences «fortement en désaccord » et «en désaccord ». Le même procédé a été utilisé pour les résultats favorables en faisant la somme des fréquences «fortement en accord » et «en accord». La catégorie "neutre» regroupe les fréquences "faiblement en accord» et «faiblement en désaccord».

Le score global de l'échelle de Lucas peut varier de 22 à 132, un score peu élevé indiquant des attitudes favorables à l'informatique, avec un score moyen de 77 . Dans la présente recherche, les scores varient entre 34 et 102, avec une moyenne de 61,12 et un écart-type de 15,4 , ce qui indique des attitudes généralement favorables à l'informatique. La consistance interne (alpha de Cronbach) est de 0,88. Les attitudes des parents à l'égard de l'informatique ont été mises en relation avec les facteurs d'âge, de sexe et de scolarité, de même qu'avec le degré de contact avec l'informatique. L'égalité des moyennes a ensuite été vérifiée au moyen d'un test de t sur les rangs à cause de la non-normalité des distributions.

Les parents plus âgés (> 33,9 ans) obtiennent un score moyen de 63,6 à l'échelle d'attitudes. Ceux dont l'âge est inférieur à la moyenne obtiennent un score moyen de 58,3. La moyenne des scores des mères est de 62,2 , celle des pères de 58,8 . Quant aux parents qui ont une 
faible scolarité, la moyenne de leurs scores d'attitudes est de 62,1 ; les parents avec une scolarité élevée obtiennent une moyenne de 59,4. Enfin, quand le degré de contact avec l'informatique est au-dessous de la moyenne, c'est-à-dire lorsque les parents ne connaissent pas la technologie, ceux-ci obtiennent une moyenne de 63,1 aux scores d'attitudes. Les parents qui ont un degré de contact avec l'informatique au-dessus de la moyenne obtiennent 56,4. Le tableau 2 présente les résultats des relations entre les attitudes et les variables indépendantes.

\section{Tableau 2}

\section{Relations entre les attitudes et les facteurs d'âge, de sexe, de scolarité et de degré de contact avec l'informatique}

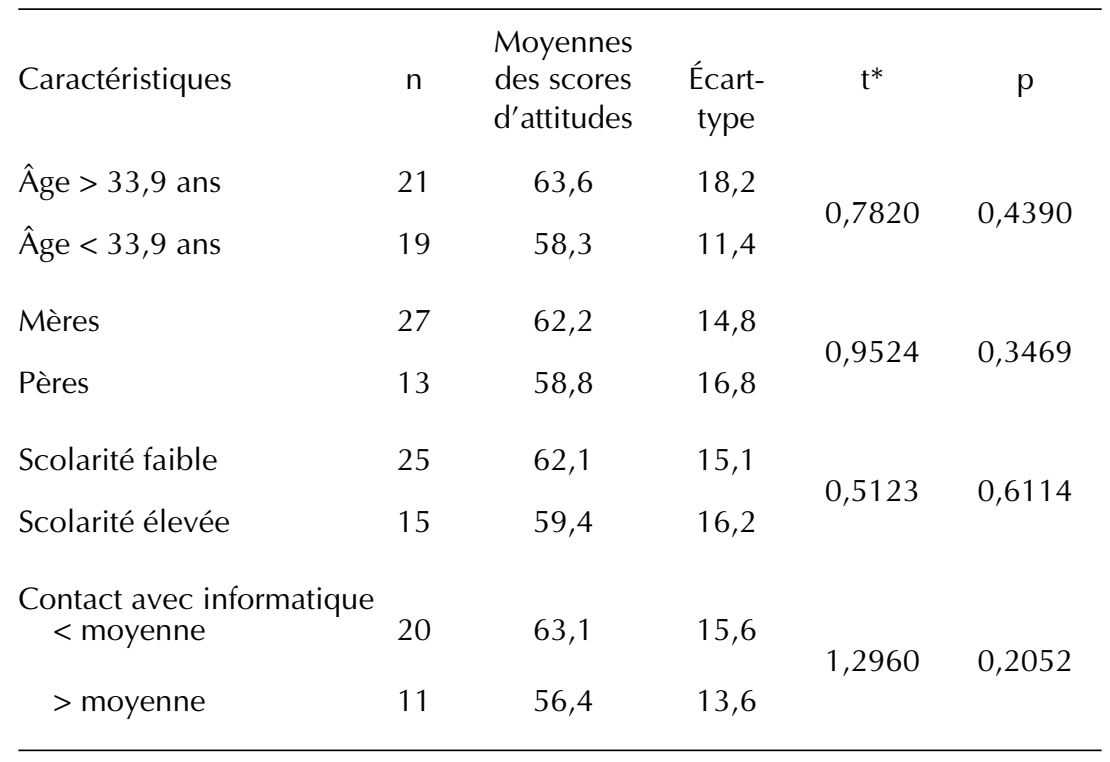

* L'égalité des moyennes a été vérifiée avec un test de t sur les rangs.

On peut conclure de cette analyse que I'attitude généralement favorable des répondants à l'égard de l'informatique n'est pas influencée de façon significative par leurs caractéristiques personnelles ni par leur degré initial de contact avec l'informatique.

\section{L'APPRÉCIATION DE L'UTILISATION DU LOGICIEL}

L'appréciation de I'utilisation du logiciel est mesurée par les réponses à un questionnaire rempli après la troisième et dernière utilisation du logiciel, et portant sur les conditions de l'expérience, l'appréciation 
de l'expérience et du contenu du logiciel ainsi que l'utilité des résultats interprétés.

L'appréciation des conditions de l'expérience (moment choisi pour passer les tests sur ordinateur; lieu où les tests étaient administrés à l'hôpital; instructions données pour utiliser l'ordinateur; temps passé à répondre aux questions; et degré de confidentialité assuré pendant la période de réponse aux questions) est complétée par une question sur la pertinence de la présence ou non d'un technicien ou d'un superviseur pendant l'expérience. Dans leur ensemble, les répondants sont satisfaits des conditions dans lesquelles s'est faite l'utilisation du logiciel. Qu'il s'agisse de l'endroit où étaient administrés les tests informatisés, des instructions données aux utilisateurs, du temps consacré à répondre aux questions ou du degré de confidentialité assuré aux répondants, le taux de satisfaction atteint $97,5 \%$ et $100 \%$ selon le cas. En ce qui concerne le moment choisi pour passer les tests, le pourcentage de satisfaction est de $80 \%$.

II n'a pas été possible de constituer un score global d'appréciation des conditions d'utilisation du logiciel, compte tenu de la faible consistance interne de la sous-échelle (alpha de Cronbach $=0,53$ ). C'est pourquoi les dernières dimensions (appréciation de l'expérience et contenu du logiciel) ont été retenues aux fins de l'analyse. Par ailleurs, 35 parents se sont prononcés sur la nécessité de la présence constante d'un technicien ou superviseur pendant l'expérience: 14 répondants $(40 \%)$ ne croient pas cette présence constante nécessaire, alors que $60 \%(n=21)$ la souhaitent.

\section{L'appréciation de l'expérience et du contenu du logiciel}

Les questions retenues ici reflètent les thèmes de préoccupation qui reviennent le plus souvent dans les écrits. Elles portent d'une part sur I'appréciation de l'expérience: la sincérité des réponses apportées lors de l'interrogation par ordinateur, la satisfaction, I'impersonnalisation des services; et, d'autre part, sur le contenu du logiciel : I'adaptation des questions posées aux situations personnelles, la correspondance des résultats communiqués aux besoins des répondants et le degré de complication lié au fait de répondre directement sur l'ordinateur. Les participants ont aussi été invités à exprimer leur motivation à poursuivre l'expérience et à formuler des commentaires sur toutes questions jugées pertinentes en rapport avec l'expérimentation du logiciel.

À la question sur la sincérité de leurs réponses à l'ordinateur, en comparaison avec une entrevue face à face avec un professionnel, $15,6 \%$ des parents $(n=5)$ ont déclaré être un peu plus sincères avec 
I'ordinateur, alors que $84,4 \%(n=27)$ ont dit être également sincères dans les deux situations. Aucun répondant ne se dit moins sincère avec l'ordinateur qu'avec un professionnel.

Le tableau 3 présente les réponses apportées aux autres questions où les parents indiquent leur degré de satisfaction sur une échelle de 1 à 5 : "tout à fait » (positions 1 et 2), "plus ou moins » (position 3) et «pas du tout» (positions 4 et 5). Les parents apprécient positivement I'expérience et le contenu du logiciel dans des proportions variant de $65 \%$ à 92,5\%. La plus faible appréciation positive, $65 \%$ des répondants, concerne la première question : «Comment avez-vous aimé votre expérience d'être interrogé par l'ordinateur?»; 22,5\% ont plus ou moins aimé et 12,5\% n'ont pas aimé du tout. Par ailleurs, $80 \%$ des parents ne sont pas du tout en accord avec l'énoncé selon lequel I'utilisation de l'ordinateur rendrait impersonnelle leur visite à l'hôpital.

TABleau 3

Appréciation de l'expérience et du contenu du logiciel

\begin{tabular}{lccccc}
\hline & \multicolumn{2}{c}{ Tout } & \multicolumn{2}{c}{ Plus } & \multicolumn{2}{c}{ Pas } \\
Questions & OBS & \multicolumn{2}{c}{ à fait } & ou moins & du tout \\
& & $n$ & $\%$ & $n$ & $\%$
\end{tabular}

\section{A) L'expérience}

Comment avez-vous aimé votre expérience d'être interrogé-e par ordinateur?

Trouvez-vous que l'utilisation de l'ordinateur rend impersonnelle votre visite

$3 \quad 7,5 \quad 5$

$12,5 \quad 32$

80,0

à l'hôpital ? *

\section{B) Contenu du logiciel}

Les questions posées vous semblent-elles adaptées à votre situation personnelle?

Trouvez-vous que les résultats qui vous ont été communiqués correspondent à vos besoins ?

Cette expérience de répondre aux questions directement à l'ordinateur vous est-elle apparue compliquée ? *

$\begin{array}{rrrrrrr}40 & 32 & 80,0 & 7 & 17,5 & 1 & 2,5 \\ 40 & 35 & 87,5 & 4 & 10,0 & 1 & 2,5 \\ 40 & 1 & 2,5 & 2 & 5,0 & 37 & 92,5\end{array}$

* Ces questions sont formulées de façon négative, mais les réponses doivent être interprétées de façon inverse. 
Les trois énoncés sur l'appréciation du contenu du logiciel (pertinence des questions posées et des résultats interprétés et facilité d'utilisation du logiciel) ont été regroupés dans une sous-échelle. La consistance interne de cette sous-échelle est de 0,80 (alpha de Cronbach) et les corrélations items-total de 0,55 à 0,79 . Le score total de cette sous-échelle peut varier de 3 à 15 ; la moyenne des scores obtenus par les parents est de 4,7, avec un écart-type de 2,1. On peut conclure de cette analyse que les parents apprécient l'expérience technologique en ce sens qu'ils sont satisfaits de la pertinence des questions posées, des résultats interprétés et de la facilité d'utilisation de l'ordinateur.

Les réponses à la question posée sur la motivation à poursuivre I'expérience dans l'avenir viennent confirmer la satisfaction des répondants. Un seul parent ne veut pas répondre à ce genre de test par ordinateur dans I'avenir, alors que 39 répondants $(97,5 \%)$ le souhaitent. Sur les 39 répondants qui veulent continuer, 44,7\% $(n=17)$ souhaitent le faire à la fréquence d'une fois par année, alors que 55,3\% $(n=21)$ veulent le faire plus d'une fois par année, selon les besoins.

Sur le plan qualitatif, douze parents ont inscrit des commentaires concernant l'expérience. Six apportent des commentaires favorables, formulent des remerciements ou des opinions, entre autres celle selon laquelle l'expérience est un bon moyen de s'analyser sans que quelqu'un porte un jugement. Quelques suggestions ont été faites: inclure des questions davantage reliées à l'âge de l'enfant, des questions reliées au stress au travail; organiser des discussions en groupe. Un parent suggère même l'absence totale du superviseur afin de préserver la pleine confidentialité de l'expérience, la disquette devenant la propriété exclusive du répondant.

\section{L'utilité des résultats interprétés}

La motivation à poursuivre l'expérience étant liée à l'appréciation faite par le parent de l'utilité des résultats qui lui sont interprétés, trois manifestations de cette utilité ont été vérifiées: réflexion, discussion et changement.

Le tableau 4 présente l'évaluation de l'utilité des résultats, établie en réponse par un oui ou un non aux questions posées. Pour 79,5\% des parents $(n=31)$ les résultats sont utiles à leur réflexion sur la situation et les comportements, alors que huit répondants (20,5\%) n'ont pas cette opinion. La discussion est une autre dimension de l'utilité: $89,5 \%(n=34)$ des répondants ont parlé des résultats avec leur conjoint ; 10,5\% $(n=4)$ ne l'ont pas fait. D'autre part, $21,4 \%(n=6)$ des 
répondants en ont parlé avec quelqu'un de leur parenté ou avec un ami ou une amie, $78,6 \%(n=22)$ ne l'ont pas fait; douze répondants $\mathrm{n}^{\prime}$ ont pas répondu à cette question, alors que les réponses manquantes aux autres items sont très rares. Un seul parent $(2,6 \%)$ a fait la demande d'une consultation professionnelle sur ses résultats.

\section{Tableau 4}

\section{Appréciation de l'utilisation des résultats}

\begin{tabular}{|c|c|c|c|c|c|}
\hline \multirow[t]{2}{*}{ Énoncés } & \multicolumn{2}{|c|}{ Oui } & \multicolumn{2}{|c|}{ Non } & Valeurs \\
\hline & $\mathrm{n}$ & $\%$ & $\mathrm{n}$ & $\%$ & manquantes \\
\hline
\end{tabular}

Utiles à la réflexion sur la situation et les comportements.

$31 \quad 79,5 \quad 8 \quad 20,5 \quad 1$

Échanges au sujet des résultats avec le conjoint.

Échanges au sujet des résultats avec un(e) parent(e) ou ami(e).

$34 \quad 89,5 \quad 4 \quad 10,5 \quad 2$

Demande d'une consultation professionnelle sur les résultats.

Contribution des résultats au changement dans la façon de voir la situation.

$\begin{array}{rrrrr}6 & 21,4 & 22 & 78,6 & 12 \\ 1 & 2,6 & 38 & 97,4 & 1 \\ 15 & 38,5 & 24 & 61,5 & 1 \\ 15 & 38,5 & 24 & 61,5 & 1\end{array}$

Contribution des résultats au changement dans les habitudes de vie ou les comportements.

\section{CONCLUSION}

Plusieurs éléments de conclusion se dégagent de la présente étude. Ils ont trait aux possibilités de l'utilisation de l'interrogation interactive avec rétroaction immédiate comme moyen d'intervention en service social auprès des parents d'enfants atteints d'une maladie chronique, telle la fibrose kystique.

Les constatations précédentes confirment, pour un échantillon de parents québécois, les conclusions de plusieurs auteurs (Stein et Reissman, 1980 ; Hofer, 1985 ; Hedlund, 1988; Hudson, 1988 ; Nurius, 1990) sur l'acceptation et les dispositions de la clientèle des services 
sociaux devant l'utilisation de l'ordinateur. Les parents qui ont participé à l'expérience de l'utilisation du logiciel Vie familiale et impact de la maladie aux deux cliniques de fibrose kystique de l'hôpital SainteJustine, à Montréal, et du CHUL, à Québec, montrent des attitudes très favorables à l'utilisation de l'informatique dans une perspective d'intervention, malgré le fait que la plupart des répondants $(64,5 \%)$ avaient peu ou presque pas de contact préalable avec l'informatique. Plus précisément, ces attitudes et leur appréciation de l'expérience s'expriment comme suit:

- Les attitudes à l'égard de l'informatique sont à peu près aussi favorables, tant chez les mères que chez les pères, peu importe leur âge, leur degré de scolarité et leur niveau de contact avec l'informatique.

- Les parents se déclarent de manière unanime très satisfaits des conditions dans lesquelles s'est déroulée l'expérience et ils expriment une appréciation très positive du contenu du logiciel quant à sa pertinence par rapport à leurs problèmes.

- Les résultats de l'expérience ont été utiles à leur réflexion et ils ont servi de base à des échanges avec leur conjoint.

Il est donc clair que l'utilisation d'un logiciel convivial tel que Vie familiale et impact de la maladie constitue, pour des parents d'enfants atteints d'une maladie chronique (la fibrose kystique), un support acceptable pour l'intervention sociale. Des études ultérieures devraient sans doute laisser voir les possibilités de transfert de cette technologie à d'autres parents vivant des situations semblables.

\section{Références bibliographiques}

ABIDIN, R.R. (1983). Parenting stress index manual. Charlottesville, VA: Pediatric Psychology Press.

BéliveAu, G. et M. Poulin (1987). Les perceptions et les attitudes des intervenants sociaux du Québec au sujet de l'utilisation de l'informatique dans leur milieu de travail. Recherche subventionnée par le Conseil de recherches en sciences humaines du Canada, Laboratoire de recherche, École de service social, Université Laval, juillet, 204 pages.

CADMAN, D., M. BOyle, P. SZATMARI et D. OfFORD (1987). "Chronic illness, disability and mental and social well-being: Findings of the Ontario child health study», Pediatrics, vol. 79, n $5: 805-813$.

CARR, A. et A. GHOSH (1983). «Response of phobic patients to direct computer assessment », British Journal of Psychiatry, vol. 142: 60-65. 
COSTEllo, B. (1982). "The application of computerized psychological and educational assessment in South Australia », School Psychology International, vol. $3: 195-202$.

ERDMAN, H.P. et S.W. FOSTER (1986). "Computer-assisted assessment with couples and families», Family Therapy, vol. 13, n' $1: 23-40$.

Erdman, H.P., J.H. Greist, M.H. Klein, J.W. Jefferson et C. Getto (1981). "The computer psychiatrist: How far have we come? Where are we heading? How far dare we go?», Behavior Research Methods and Instrumentation, vol. 13, $\mathrm{n}^{\circ} 4: 393-398$.

ERDMAN, H.P., J.H. GREIST, M.H. KLEIN (1985). "Direct patient computer interviewing ", Journal of Consulting and Clinical Psychology, vol. 53, $\mathrm{n}^{\circ} 6: 760-773$.

GRAWITZ, M. (1986). Lexique des sciences sociales. France: Éditions Dalloz.

HEDLUND, J.L. (1988). "Mental health computing: Directions for research», Computers in Human Services, vol. 2, $\mathrm{n}^{\circ}$ 3-4: 9-36.

HEDLUND, J.L., B.W. VIEWEG et D.N. CHO (1985). "Mental health computing in the 1980's: II. Clinical applications », Computers in Human Services, vol. 1, $\mathrm{n}^{\circ} 2: 1-31$.

HOFER, P.J. (1985). "Developing standards for computerized psychological testing », Computers in Human Behavior, vol. 1 : 301-315.

HOFER, P.J. et B.F. GREEN (1985). "The challenge of competence and creativity in computerized psychological testing », Journal of Consulting and Clinical Psychology, vol. 53, $\mathrm{n}^{\circ} 6: 826-838$.

HUDSON, W. H., (1988). "Measuring clinical outcomes and their use for managers ", Administration in social work, vol. 11, $\mathrm{n}^{\circ}$ 3-4 : 59-71.

JOHNSON, J.H. et T.A. WILLIAMS (1980). Using on-line computer technology to improve services response and decision-making effectiveness in a mental health admitting system, dans J.B. Sidowski, J.H. Johnson et T.A. Williams (dir.). Technology in mental health care delivery systems. Norwood, NJ: Ablex, p. 237-249.

JOHNSON, J.H., S.W. GODIN et M.L. BLOOMQUIST (1981). "Human factors engineering in computerized mental health care delivery », Behavior Research Methods and Instrumentation, vol. 13, $\mathrm{n}^{\circ} 4:$ 425-429.

KLINGER, D.E., J.H. JOHNSON et T.A. WILLIAMS (1976). "Strategies in the evaluation of an on-line computer-assisted unit for intake assessment of mental health patients", Behavior Research Methods and Instrumentation, vol. 8, no $2: 94-100$.

LEMYRE, L. (1986). Stress psychologique et appréhension cognitive. Thèse de doctorat (inédite), École de psychologie, Université Laval.

LEMYRE, L., R. TESSIER et L. FILLION (1990). Mesure de stress psychologique: manuel d'utilisation. Behaviora.

LOCKE, H.J. et K.M. WALLACE (1959). "Short marital adjustment and prediction tests: their reliability and validity », Marriage and Family Living, vol. 21, $\mathrm{n}^{\circ} 2: 251-255$.

LOGIE, A.R., J.A. MADIRAZZA et I.W. WEBSTER (1976). "Patient evaluation of a computerized questionnaire», Computers and Biomedical Research, vol. 9: 169-176. 
LUCAS, R.S. (1977). "A study of patients attitudes to computer interrogation. International », Journal of Man-machine Studies, vol. 9: 69-86.

MATHISEN, K.S. (1988). "Issues in research on clinical computers applications for mental health », Computers in Human Services, vol. 2, $\mathrm{n}^{\circ}$ 3-4 : 87-107.

MERRELL, K.W. (1985). "Computer use in psychometric assessment: evaluating benefits and potential problems », Computers in Human Services, vol. 1, $\mathrm{n}^{\circ} 3: 59-67$.

MEZZICH, J.E., J.T. DOW et G.A. COFFMAN (1982). "Developing an efficient clinical information system for a comprehensive psychiatric institute: I. Principles, design and organization », Behavior Research, Methods and Instrumentation, vol. 13, $\mathrm{n}^{\circ} 4: 459-463$.

MoOs, R. (1974). Systems for the assessment and classification of human environments : the overview, dans R. Moss et P. Insel (dir.). Issues in social ecology: human milieus. Polo Alto: Mayfield, p. 5-28.

NURIUS, P. (1990). "A review of automated assessment", Computers in Human Services, vol. 6 , $\mathrm{n}^{\circ} 4: 265-281$.

Poulin, M. et al. (1991). Les effets et l'appréciation de l'utilisation d'un logiciel interactif par les parents d'enfants atteints de fibrose kystique. Rapport final, recherche subventionnée par la Fondation canadienne de fibrose kystique, Laboratoire de recherche, École de service social, Université Laval, 30 novembre, 300 pages.

Poulin, M., A. BEAudoin et F. TURGEON-KRAWCZUK (1992). "État des connaissances à l'origine de l'utilisation du logiciel Vie familiale et impact de la maladie: attitudes et motivation de la clientèle et préoccupations des professionnels", Service social, vol. 41, n $3: 105-123$.

SAMPSON, J.P. (1983a). "Measurement forum. Computer-assisted testing and assessment: current status and implications for the future », Measurement and Evaluation in Guidance, vol. 15, $\mathrm{n}^{\circ} 3$ : 293-299.

SAMPSON, J.P. (1983b). "An integrated approach to computer implications in counseling psychology », The Counseling Psychologist, vol. 11, $\mathrm{n}^{\circ} 4$ : 65-74.

SiEGEL, C. et M.J. AleXANDER (1988). "Research activities and their methodologies in mental health computing ", Computers in Human Services, vol. 2, $\mathrm{n}^{\circ}$ 3-4: 61-83.

SKINNER, H.A. et B.A. ALLEN (1983). "Does the computer make a difference? Computerized versus face-to-face versus self-report assessment of alcohol, drug and tobacco use », Journal of Consulting and Clinical Psychology, vol. 51, $\mathrm{n}^{\circ} 2:$ 267-275.

SKINNER, H. et P. STEINHAUER (1983). "The family assessment measure », Canadian Journal of Community Mental Health, vol. 2, $\mathrm{n}^{\circ} 2:$ 91-105.

SPACE, L.G. (1981). "The computer as a psychometrician», Behavior Research, Methods and Instrumentation, vol. 13, n $4:$ 595-606.

STEIN, R. et C. REISSMAN (1980). "The development of an impact on family scale: Preliminary findings », Medical Care, vol. 18, $\mathrm{n}^{\circ} 4:$ 465-472.

TURGEON-KRAWCZUK, F. (1991). Un logiciel interactif en service social: effets sur les parents d'enfants atteints d'une maladie chronique. Thèse de doctorat, présentée à la Faculté des études supérieures, Université Laval, novembre, 282 pages. 


\section{AN NEXE}

\section{Attitudes des parents à l'égard de l'informatique}

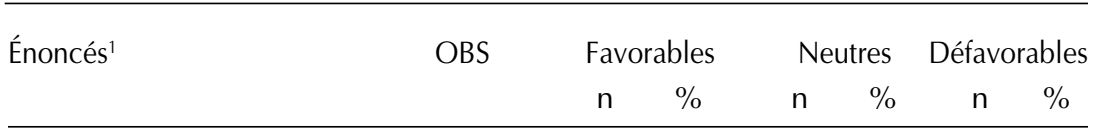

1. ${ }^{*}$ L'ordinateur ne sera jamais capable de remplacer l'équipe médicale pour faire une partie du travail.

$\begin{array}{lllllll}39 & 12 & 30,8 & 10 & 25,6 & 17 & 43,6 \\ 40 & 22 & 55,0 & 9 & 22,5 & 9 & 22,5 \\ & & & & & & \\ 40 & 20 & 50,0 & 11 & 27,5 & 9 & 22,5 \\ 40 & 26 & 65,0 & 9 & 22,5 & 5 & 12,5 \\ 40 & 28 & 70,0 & 7 & 17,5 & 5 & 12,5\end{array}$

2. Si un ordinateur peut poser des questions à la place de l'équipe, les professionnels passeront plus de temps à penser aux réponses des bénéficiaires.

3. Finalement, les gens deviendront les esclaves des machines.

4.* Si l'on utilise beaucoup les ordinateurs nous devrons bientôt payer pour aller à l'hôpital.

5. Même si je suis interrogé(e) par un ordinateur, il me semble que quelqu'un fait attention à mes réponses.

6. Les ordinateurs me rendent moins nerveux ou nerveuse que les professionnels de l'équipe.

7. La qualité des soins s'améliorera dans les hôpitaux si on se sert beaucoup des ordinateurs.

8. ${ }^{*}$ Autant aller chez le pharmacien chercher conseil que de s'embêter à aller dans un hôpital qui utilise un ordinateur.

9.* Les ordinateurs ne peuvent nous faire que du tort s'ils sont très utilisés dans les hôpitaux.

10. Plus de personnes pourront consulter les médecins spécialistes si l'on fait grand usage des ordinateurs à l'hôpital.

20

50,0




\begin{tabular}{|c|c|c|c|c|c|c|}
\hline \multirow[t]{2}{*}{ Énoncés ${ }^{1}$} & \multirow[t]{2}{*}{ OBS } & \multicolumn{2}{|c|}{ Favorables } & \multicolumn{2}{|c|}{ Neutres } & Défavorables \\
\hline & & $\mathrm{n}$ & $\%$ & $\mathrm{n}$ & $\%$ & $\%$ \\
\hline
\end{tabular}

11.* Les membres de l'équipe négligeront davantage

40

les bénéficiaires si les ordinateurs

$\begin{array}{llllll}26 & 65,0 & 12 & 30,0 & 2 & 5,0\end{array}$

leur viennent en aide.

12. On assurera un traitement de première qualité à la plupart des gens si l'on utilise beaucoup les ordinateurs dans les hôpitaux.

40

$20 \quad 50,0 \quad 20 \quad 50,0 \quad 0 \quad-$

13. La qualité des soins s'améliorera certainement si on utilise beaucoup les ordinateurs à l'hôpital.

14.* Les membres de l'équipe seront moins dévoués aux bénéficiaires si les ordinateurs les aident.

$\begin{array}{llllll}24 & 60,0 & 9 & 22,5 & 7 & 17,5\end{array}$

15.* Les ordinateurs rendront les relations entre l'équipe et les bénéficiaires plus difficiles qu'elles ne le sont actuellement.

16. La plupart des bénéficiaires tireront profit de la grande précision des ordinateurs.

17.* On ne devrait pas avoir confiance dans les ordinateurs quand il s'agit de la santé.

18.* Si les ordinateurs sont très utilisés dans les hôpitaux, ce sera plus difficile d'obtenir des soins médicaux à la maison.

19.* II sera plus difficile aux gens âgés de répondre aux ordinateurs.

20. Les ordinateurs dans les hôpitaux feront gagner du temps à tout le monde.

21. On se sent plus à l'aise de s'asseoir devant un ordinateur que de s'asseoir devant un membre de l'équipe.

22. Plus de gens obtiendront un traitement d'experts si on utilise beaucoup les ordinateurs à l'hôpital. 